\title{
Pratiques enseignantes et vécus professionnels en période de crise sanitaire en Belgique francophone
}

Natacha Duroisin, Romain Beauset, Laurie Simon et Chloé Tanghe

\section{OpenEdition}

Édition électronique

URL : https://journals.openedition.org/ries/10304

DOI : 10.4000/ries. 10304

ISSN : 2261-4265

Éditeur

France Education international

\section{Édition imprimée}

Date de publication : 1 avril 2021

Pagination : $27-30$

ISBN : 978-2-85420-629-6

ISSN : $1254-4590$

Référence électronique

Natacha Duroisin, Romain Beauset, Laurie Simon et Chloé Tanghe, «Pratiques enseignantes et vécus professionnels en période de crise sanitaire en Belgique francophone », Revue internationale d'éducation de Sèvres [En ligne], 86 | avril 2021, mis en ligne le 01 avril 2022, consulté le 24 mars 2023. URL : http://journals.openedition.org/ries/10304 ; DOI : https://doi.org/10.4000/ries.10304

Ce document a été généré automatiquement le 24 mars 2023.

Tous droits réservés 


\title{
Pratiques enseignantes et vécus professionnels en période de crise sanitaire en Belgique francophone
}

\author{
Natacha Duroisin, Romain Beauset, Laurie Simon et Chloé Tanghe
}

1 Dans le contexte de crise sanitaire, nous avons, depuis le début du confinement, investigué les pratiques des enseignants belges francophones par le biais d'enquêtes quantitatives, menées en ligne, sur des échantillons de convenance. Complétées par des enseignants du maternel, primaire et secondaire, ces enquêtes ont pour principal objectif de fournir un état des lieux de la situation d'enseignement-apprentissage à différents moments de la pandémie, en vue de comprendre la perception de la situation par les enseignants en Fédération Wallonie-Bruxelles (FWB).

2 Dans cet article, les principaux résultats de la deuxième enquête sont présentés ${ }^{1}$. Celleci a été réalisée en octobre 2020, après la rentrée scolaire, sur un échantillon de 518 enseignants du maternel, du primaire et du secondaire ${ }^{2}$.

\section{Le contexte belge en période de crise sanitaire}

3 Le Conseil national de sécurité (CNS) constitué du gouvernement fédéral et des entités fédérées a décidé de suspendre, à partir du 16 mars 2020, les cours en présentiel dans toutes les écoles de la FWB afin de respecter les mesures de confinement liées à la crise sanitaire de la Covid-19. Plusieurs circulaires ont alors été rédigées à l'intention des acteurs des établissements scolaires, leur transmettant règles et recommandations. Si, pendant les premiers jours suivant la décision du CNS, il n'a pas été demandé aux enseignants de prévoir du travail à distance pour les élèves (circulaire 7508), la circulaire 7515, du 17 mars 2020, informait les enseignants de la possibilité d'envoyer des travaux à réaliser à domicile aux élèves, à condition que ceux-ci portent sur des contenus abordés préalablement en classe et soient uniquement évalués de manière formative. À la suite de la présentation d'un plan de déconfinement et conformément à la circulaire $7550 \mathrm{du} 25$ avril 2020, la réouverture des écoles pouvait s'effectuer en deux 
phases. À partir du 18 mai 2020 , les élèves de $6^{e}$ primaire et ceux de dernière année du secondaire $\left(6^{\mathrm{e}}\right.$ ou $\left.7^{\mathrm{e}}\right)$ pouvaient reprendre les cours en présentiel, par groupe et à concurrence de deux jours par semaine maximum. À partir du 25 mai 2020, d'autres élèves pouvaient reprendre le chemin de l'école, en groupe également: un jour maximum pour les élèves de $1^{\text {re }}$ primaire et si possible de $2^{\mathrm{e}}$ primaire et deux jours maximum pour les élèves de $2^{\mathrm{e}}$ secondaire. Pour tous les élèves, le gouvernement a annulé les épreuves externes certificatives (CEB, CE1D et CESS). Enfin, la circulaire 7599 (27 mai 2020) annonçait la reprise des cours en présentiel à temps plein, dès le 2 juin, de toutes les classes de l'enseignement maternel et, dès le 8 juin, de toutes celles de l'enseignement primaire. Aucun changement n'a été annoncé concernant l'enseignement secondaire.

4 La rentrée de septembre s'est opérée en "code jaune" pour tous les niveaux d'enseignement; l'obligation scolaire a donc été pleinement rétablie dans le respect de différentes mesures sanitaires. Pour l'enseignement primaire, le port du masque et la distanciation sociale étaient obligatoires pendant l'activité pour les enseignants uniquement, alors que pour l'enseignement secondaire, ces mesures étaient obligatoires pendant l'activité, pour les élèves comme pour le personnel. Les circulaires 7686 (18 août 2020) et 7691 (19 août 2020) mentionnaient que les établissements scolaires devaient développer une stratégie de différenciation et de lutte contre le décrochage. Il y était stipulé que la priorité des écoles devait être de (re)créer du lien social entre les élèves, entre les membres du personnel et entre les élèves et les membres du personnel, de (re)créer un cadre bienveillant et un climat propice à la reprise des apprentissages et d'assurer un accompagnement adéquat à chaque élève, en particulier aux élèves qui risquaient de rencontrer des difficultés les empêchant de suivre le rythme scolaire. Malgré cette rentrée en code jaune, il a été demandé aux établissements primaires et secondaires de préparer une stratégie d'hybridation des apprentissages (enseignement partiellement à distance ou en présentiel) en cas de mesures de mises en quatorzaine pour le fondamental et de passage en "code orange» ou "code rouge » pour le secondaire. La circulaire 7725 (3 septembre 2020), présentait, quant à elle, des ressources dans le cadre de la stratégie de la rentrée 2020-2021. Parmi ces ressources, un document identifiait les savoirs, savoir-faire et compétences essentiels parmi ceux définis dans les référentiels en vigueur ${ }^{3}$.

\section{Le ressenti des enseignants belges face au contexte de crise sanitaire}

5 Les enseignants ont été questionnés sur leurs perceptions à l'égard de la situation au début de l'année scolaire 2020-2021. Un retour à l'enseignement en présentiel était plébiscité par $77,3 \%$ des enseignants répondants; $19,4 \%$ auraient préféré un enseignement hybride et seuls 3,3\% souhaitaient reprendre les cours à distance. 88,7\% des enseignants interrogés étaient enthousiastes à l'idée de reprendre les cours en présentiel malgré la peur, pour plus de la moitié des répondants (50,8\%), d'être contaminés et un stress plus ou moins important, étant donné les conditions sanitaires, pour $56,1 \%$ des enseignants. Quand l'enseignement s'est déroulé en présentiel, 68,5\% des enseignants ont déclaré avoir dû faire face à des taux d'absentéisme plus importants que les années précédentes ainsi qu'à des élèves démotivés, suite au 
confinement. En outre, $92 \%$ des enseignants estimaient que la période de confinement avait amplifié les inégalités scolaires et 53,4 \% que l'écart entre les élèves plus faibles et plus forts s'était creusé, cet écart étant visible dès la rentrée. Ainsi, $83,4 \%$ des enseignants ont remarqué une baisse de niveau des apprenants étant donné le confinement. Parmi ces derniers, $62,7 \%$ estimaient que cette baisse était relative, c'està-dire variable d'un élève à l'autre.

Concernant le port du masque, $72,1 \%$ des répondants (parmi lesquels $81,7 \%$ d'enseignants du primaire et $66,9 \%$ d'enseignants du secondaire) estimaient que celuici était très contraignant pour enseigner; $77,4 \%$ des enseignants du secondaire estimaient que le port du masque par leurs élèves les rassurait, alors que 22,6\% affirmaient le contraire. $48,8 \%$ des enseignants ont estimé que le port du masque par les élèves était problématique.

\section{Quelles priorités en début d'année scolaire pour les enseignants?}

7 Les répondants ont ordonné par importance décroissante six priorités qui leur étaient proposées dans le questionnaire : la motivation des élèves, les apprentissages scolaires, l'attention, la méthodologie de travail, la gestion du stress et pour finir, la préparation des élèves à l'utilisation d'outils numériques permettant l'enseignement-apprentissage à distance.

8 La faible importance accordée à l'anticipation d'un éventuel reconfinement s'illustre également par les réponses des enseignants à d'autres questions. En effet, plus de la moitié $(54,1 \%)$ ont affirmé ne pas avoir préparé les élèves à l'enseignement à distance. Par ailleurs, de nombreux enseignants ont indiqué ne pas s'être renseignés sur les équipements numériques de leurs nouveaux élèves depuis la rentrée et seuls $16,6 \%$ ont admis connaître parfaitement les équipements de chacun de leurs élèves. L'utilisation d'une plateforme en ligne, pourtant prescrite dans les circulaires, n'était mise en œuvre que par $43,6 \%$ des répondants.

\section{Quelles pratiques ont émergé en début d'année scolaire pour les enseignants?}

9 La crise sanitaire semble avoir fait émerger de nouvelles pratiques qui s'avèrent être cohérentes avec les priorités évoquées. Près de $60 \%$ des enseignants ont admis avoir changé leurs habitudes lors de la rentrée. 70,3\% d'entre eux ont, par exemple, jugé nécessaire de laisser du temps aux élèves pour qu'ils puissent exprimer leurs émotions face à la situation sanitaire. Le rappel des gestes barrières, parfois plusieurs fois par jour, est apparu comme un nouvel élément de la pratique enseignante pour $88,2 \%$ des enseignants.

10 Concernant les apprentissages scolaires, des actions ont été entreprises par certains enseignants afin de pallier d'éventuels retards dans les apprentissages ${ }^{4}$. C'est le cas, par exemple, de la focalisation sur les apprentissages jugés les plus importants $(48,3 \%)$ ou de l'utilisation de dossiers spécifiques pour un travail autonome (24,8\%). Par ailleurs, à la rentrée, $41,9 \%$ des enseignants ont réalisé un rappel des thématiques les plus importantes de l'année passée, $38,6 \%$ se sont concentrés sur les apprentissages 
«essentiels », 38,4\% ont débuté l'année par la matière non vue l'année précédente, $38,2 \%$ ont débuté par des exercices de remise à niveau. Moins d'un tiers des répondants (29,3\%) ont débuté l'année directement par de nouveaux contenus et $15,3 \%$ des enseignants interrogés ont déclaré n'avoir rien mis en place pour gérer les retards. Concernant les pratiques d'évaluation, on observe une tendance à l'évaluation formative $(78,5 \%)$ ou diagnostique $(57,2 \%)$, plus qu'aux évaluations sommatives et certificatives (respectivement 40,5\% et $21,0 \%$ ). Enfin, 33,2\% des enseignants interrogés ont proposé une ou plusieurs évaluations dès la rentrée.

11 L'impact des mesures sanitaires sur l'enseignement-apprentissage a également été interrogé : il en ressort que $77,3 \%$ des enseignants estiment que leurs pratiques et comportements ont été impactés. Parmi les changements relevés, $62,5 \%$ des répondants ont jugé que la distanciation sociale et le port du masque les avaient contraints à davantage d'enseignement magistral. Notons que $78,9 \%$ des enseignants ont déclaré impossible le respect de la distanciation sociale au vu du nombre d'élèves par classe et de la taille de celle-ci.

12 Enfin, 42,5\% des répondants ont déclaré avoir conservé à la rentrée scolaire certaines habitudes prises durant le confinement. Les plus citées sont la mobilisation de ressources en ligne, la mise en ligne des séances de cours, une communication accrue avec les familles, la mise en place de meilleures pratiques de différenciation ou encore la diminution des pratiques d'évaluation. Si certains enseignants ont dit avoir poursuivi l'utilisation d'outils numériques découverts durant le confinement, ce n'était pas le cas pour plus de la moitié d'entre eux $(57,5 \%)$ et ce pour diverses raisons (manque de temps, manque d'intérêt, manque de maîtrise, manque de matériel...).

\section{Et après ?}

13 Si l'enquête a permis d'identifier les perceptions des enseignants ainsi que leurs priorités et pratiques depuis le début de l'année scolaire, se pose à présent la question de la persistance de ces nouvelles pratiques au-delà de la crise sanitaire. Ces questions constituent l'une des préoccupations majeures de notre équipe de recherche. Des entretiens qualitatifs ainsi qu'une troisième enquête à large échelle sont envisagés.

\section{NOTES}

1. Les auteurs renvoient à leur rapport de recherche pour davantage de précisions concernant les résultats : Duroisin N., Beauset R., Flamand A. et Leclercq M. (2021). École \& Covid. Pratiques enseignantes en temps de pandémie (résultats de la deuxième enquête). Rapport de recherche. Université de Mons, Belgique. En ligne : www.CAPTE.be

2. Parmi les 911 personnes ayant accepté de participer à cette enquête, n'ont ici été retenus dans l'échantillon que les questionnaires complétés à plus de $50 \%$ par des enseignants de l'enseignement maternel, primaire et secondaire de la FWB (tous réseaux confondus), soit 
518 questionnaires. La durée estimée pour compléter l'enquête était de 10 à 15 minutes. Les chercheurs ont été particulièrement attentifs à la protection des données des répondants.

3. Les essentiels et balises diagnostiques pour la rentrée 2020 sont accessibles sur: http:// enseignement.be/download.php?do_id=15922/

4. Pour cette question, les enseignants pouvaient mentionner plusieurs de ces actions.

\section{INDEX}

Palabras claves : crisis sanitaria, práctica profesional, docente, escuela preescolar, escuela primaria, escuela secundaria, política educacional

Keywords : health crisis, professional practice, teachers, nursery schools, primary schools, secondary schools, educational policy

Mots-clés : crise sanitaire, pratique professionnelle, enseignant, école maternelle, école primaire, école secondaire, politique éducative

Index géographique : Belgique francophone

\section{AUTEURS}

\section{NATACHA DUROISIN}

Natacha Duroisin, Ph. D, est professeure à l'Université de Mons en Belgique. Ses champs d'expertise concernent la formation enseignante et la (neuro)psychologie des apprentissages scolaires. Elle dirige la recherche sur les pratiques enseignantes en temps de pandémie ainsi que d'autres recherches menées dans le cadre du Pacte pour un enseignement d'excellence (notamment un projet pilote sur la « Différenciation des apprentissages et l'accompagnement personnalisé ») et est impliquée dans le cadre de la Réforme de la formation initiale des enseignants en Fédération Wallonie-Bruxelles. Courriel : natacha.duroisin[at]umons.ac.be

\section{ROMAIN BEAUSET}

Romain Beauset est titulaire d'un master en sciences de l'éducation. Doctorant sous mandat Aspirant FRS-FNRS à l'Université de Mons, il mène une recherche sur le développement des habiletés spatiales chez les enfants de 6 à 14 ans et participe également à la recherche sur les pratiques enseignantes en temps de pandémie. Courriel : romain.beauset[at]umons.ac.be

\section{LAURIE SIMON}

Laurie Simon est titulaire d'un master en sciences de l'éducation. Après avoir travaillé en tant qu'enseignante dans le primaire, elle est actuellement assistante de recherche à l'Université de Mons. Elle participe au projet pilote « Différenciation et accompagnement personnalisé » dans le secondaire et porte un intérêt particulier aux pratiques enseignantes associées à la notion de bien-être. Courriel : laurie.simon[at]umons.ac.be. 


\section{CHLOÉ TANGHE}

Chloé Tanghe est titulaire d'un master en sciences biomédicales et détient une formation pédagogique lui permettant d'enseigner les sciences dans le secondaire supérieur. Elle est actuellement assistante de recherche à l'Université de Mons et participe au projet pilote « Différenciation et accompagnement personnalisé » dans le secondaire. Courriel :

chloe.tanghe[at]umons.ac.be 